\title{
SHIODA-TATE FORMULA FOR AN ABELIAN FIBERED VARIETY AND APPLICATIONS
}

\author{
KeiJi Oguiso
}

\begin{abstract}
We give an explicit formula for the Mordell-Weil rank of an abelian fibered variety and some of its applications for an abelian fibered hyperkähler manifold. As a byproduct, we also give an explicit example of an abelian fibered variety in which the Picard number of the generic fiber in the sense of scheme is different from the Picard number of generic closed fibers.
\end{abstract}

\section{Introduction}

The aim of this note is to give an explicit formula for the Mordell-Weil rank of an abelian fibered variety (Theorem 1.1) and some of its applications for an abelian fibered hyperkähler manifold (Theorem 3.1). Our formula is a formal generalization of the famous formula for an elliptic surface due to Shioda and Tate $([20,21,22])$ and some formula for a Calabi-Yau fiber space by Kawamata [11]. Our formula is also very close to a result of Bruno Kahn [9], which was recently posted on the ArXiv.

For the precise statement, we introduce a few notations. By an abelian fibered variety, we mean a proper surjective morphism $\varphi: X \longrightarrow Y$ with rational section $O$, from a normal projective variety $X$ to a normal projective variety $Y$ such that the generic fiber $A:=X_{\eta}$ is a positive-dimensional abelian variety defined over $\mathbb{C}(Y)$ (with origin $O$ ). Here, by a rational section, we mean a subvariety $S \subset X$ such that $\varphi \mid S: S \longrightarrow Y$ is a birational morphism. We assume that:

(1) $X$ has only $\mathbb{Q}$-factorial rational singularities;

(2) $Y$ has only $\mathbb{Q}$-factorial rational singularities;

(3) $\varphi$ is equi-dimensional in codimension 1 in the sense that there is no prime divisor $D$ on $X$ such that $\operatorname{dim} \varphi(D) \leq \operatorname{dim} Y-2$, and

(4) $h^{1}\left(X, \mathcal{O}_{X}\right)=h^{1}\left(Y, \mathcal{O}_{Y}\right)$

Let $K=\mathbb{C}(Y)$. The Mordell-Weil group $\operatorname{MW}(\varphi)$ is, by definition, the group $A(K)$ of $K$-rational points of $A$. In more geometric terms, $\mathrm{MW}(\varphi)$ is a group of

Received March 20, 2007.

2000 Mathematics Subject Classification. 14C22, 14D06, 14J28, 14J40.

Key words and phrases. Mordell-Weil group.

The author was supported by JSPS. 
rational sections of $\varphi$, which we can naturally regarded as an abelian subgroup of $\operatorname{Bir}(X)$, the group of the birational transformations of $X$. The Néron-Severi group NS $\left(A_{K}\right)$ of $A$ is the group of algebraic equivalence classes of divisors defined over $K$.

Let $\Delta \subset Y$ be the discriminant divisor of $\varphi$ and let $\Delta=\cup_{i=1}^{k} \Delta_{i}$ and $\varphi^{*}\left(\Delta_{i}\right)_{\text {red }}=\cup_{j=0}^{m_{i}-1} D_{i j}$ be the irreducible decomposition of $\Delta$ and $\varphi^{*}\left(\Delta_{i}\right)_{\text {red }}$. Note that $m_{i}$ is the number of prime divisors on $X$ lying over $\Delta_{i}$.

Our main result is as follows:

Theorem 1.1. Under the assumption (1)-(4) and the notation above, the Mordell-Weil group $\mathrm{MW}(\varphi)$ is a finitely generated abelian group of rank

$$
\operatorname{rank} \operatorname{MW}(\varphi)=\rho(X)-\rho(Y)-\operatorname{rank} N S\left(A_{K}\right)-\sum_{i=1}^{k}\left(m_{i}-1\right) .
$$

Here $\rho(X)$ (resp. $\rho(Y))$ is the Picard number of $X$ (resp. of $Y)$. In particular,

$$
\operatorname{rank} \operatorname{MW}(\varphi) \leq \rho(X)-2 .
$$

We believe that our assumptions (1)-(3) are not too restrictive in the view of minimal model theory in higher dimension. In our formulation, some condition like (4) is necessary for finite generation. For instance, in the product case $\mathrm{pr}_{2}: A \times Y \longrightarrow Y$, the Mordell-Weil group $\mathrm{MW}\left(\mathrm{pr}_{2}\right)$ is far from being finitely generated. See [9] for a more unified treatment including such cases.

Theorem 1.1 is proved in Section 2. An application for an abelian fibered hyperkähler manifold will be given in Section 3 (Theorem 3.1). This part was inspired by a series of work of Matsushita [13, 14, 15], a work of Sawon [18] and also by the author's previous work [17]. As one of byproducts of our Theorem 1.1, we shall give an explicit example of an abelian fibered hyperkähler manifold $f: X \longrightarrow \mathbb{P}^{d}$ of dimension $2 d \geq 4$ such that all the smooth fibers $X_{t}$ are isomorphic to $E_{\zeta}^{d}$, the self-product of the elliptic curve $E_{\zeta}$ of period $\zeta=e^{2 \pi i / 3}$, so that $\rho\left(X_{t}\right) \geq 2$, but $\operatorname{rankNS}\left(A_{K}\right)=1$ for the generic fiber $A_{K}$ in the sense of scheme (Theorem 3.1(2)). This example shows that the Picard number of the generic fiber in the sense of scheme is not always the same as the Picard number of generic closed fibers. It might be interesting to compare this with Example 3.5, a more "moderate" example.

Acknowledgement. An initial idea of this note has been grown up during my stay at KIAS August 2005 and finalized in the present form at KIAS March 2007. I would like to express my thanks to Professors B. Kahn, F. Campana, J. Keum, B. Kim, J. M. Hwang, J. Sawon, D.-Q. Zhang for several valuable discussions. After acceptance of this note, I was also informed from Amilcar Pacheco that they have also obtained a similar result when the source and target are both smooth [8]. 


\section{Proof of Theorem 1.1}

In this section, we shall prove Theorem 1.1.

By $Z(X)$ (resp. $N(X)$ ), we denote the free abelian group of Weil divisors on $X$ (resp. the ablelian group of the linear equivalence classes of Weil divisors on $X$ ). By Div $X$, we denote the free abelian group of Cartier divisors on $X$. Pic $X$ is nothing but the group of linear equivalence classes of Cartier divisors. Since $X$ is normal and projective, we have a natural inclusion, $\operatorname{Pic} X \subset N(X)$.

Let us start with the following lemma due to Kawamata [10]:

Lemma 2.1. The quotient group $N(X) / \operatorname{Pic} X$ is a finite abelian group.

Proof. Let $\pi: X^{\prime} \longrightarrow X$ be a resolution of singularities of $X$. By [10, Proof of Lemma (1.1)], Pic $X^{\prime} / \pi^{*} \operatorname{Pic} X$ is a finitely generated abelian group. Note that Pic $X^{\prime}=N\left(X^{\prime}\right)$, because $X^{\prime}$ being smooth. Thus, the natural surjective homomorphism $N\left(X^{\prime}\right) \longrightarrow N(X)$, together with the projection formula, induces a surjective homomorphism $\operatorname{Pic} X^{\prime} / \pi^{*} \operatorname{Pic} X \longrightarrow N(X) / \operatorname{Pic} X$. Therefore $N(X) / \mathrm{Pic} X$ is also a finitely generated abelian group. On the other hand, $N(X) /$ Pic $X$ is a torsion group, because $X$ is $\mathbb{Q}$-factorial. Combining these two, we obtain the result.

Lemma 2.2. The quotient group $\operatorname{Pic} X / \varphi^{*} \operatorname{Pic} Y$ is a finitely generated abelian group of rank $\rho(X)-\rho(Y)$.

Proof. Since $\varphi_{*} \mathcal{O}_{X}=\mathcal{O}_{Y}$, the homomorphism $\varphi^{*}$ is injective. So is its restriction $\left(\varphi^{*}\right)^{0}: \operatorname{Pic}^{0} Y \longrightarrow \operatorname{Pic}^{0} X$. Here $\operatorname{Pic}^{0} Y$ (resp. $\operatorname{Pic}^{0} X$ ) is an abelian variety of dimension $h^{1}\left(\mathcal{O}_{Y}\right)$ (resp. $h^{1}\left(\mathcal{O}_{X}\right)$ ). This is because $Y$ (resp. $X$ ) has only rational singularities. Since $h^{1}\left(\mathcal{O}_{Y}\right)=h^{1}\left(\mathcal{O}_{X}\right)$, the homomorphism $\left(\varphi^{*}\right)^{0}$ is then an isomorphism. Thus, $\varphi^{*}: \mathrm{NS}(Y) \longrightarrow \mathrm{NS}(X)$ is an injective homomorphism and $\operatorname{Pic} X / \varphi^{*} \operatorname{Pic} Y \simeq \operatorname{NS}(X) / \varphi^{*} \mathrm{NS}(Y)$. This implies the result.

From now, we need some elementary properties of abelian varieties defined over non-closed fields, for which we refer to the reader an excellent account of Milne in [6, Chapter V].

Let Pic $A_{K}$ be the Picard group of $A=A_{K}$, i.e., the group of linear equivalence classes of divisors defined over $K$, and let $c:$ Pic $A_{K} \longrightarrow \mathrm{NS}\left(A_{K}\right)$ be the natural map. By definition, $c$ is a surjective homomorphism and its kernel is the group $\mathrm{Pic}^{0} A_{K}$, i.e., the group of divisors defined over $K$, which are algebraically equivalent to 0 modulo linearly equivalent to 0 . We have $\operatorname{Pic}^{0} A_{K}=\hat{A}_{K}(K)$, where $\hat{A}_{K}$ is the dual abelian variety of $A=A_{K}$, both of which are defined over $K$.

Let $D$ be a Weil divisor on $X$. Then $D \mid X \backslash \operatorname{Sing} X$ is Cartier, and therefore, we have a natural surjective homomorphism:

$$
r_{N(X), A}: N(X) \longrightarrow \operatorname{Pic} A_{K} ; \operatorname{cl}(D) \mapsto \operatorname{cl}(D \mid A) .
$$


Here $\operatorname{cl}(D)$ is the linear equivalence class of $D$. Since $r_{N(X), A}\left(\varphi^{*} \operatorname{Pic} Y\right)=\{0\}$, the homomorphism $r_{N(X), A}$ induces a homomorphism

$$
r_{A}: \operatorname{Pic} X / \varphi^{*} \operatorname{Pic} Y \longrightarrow \operatorname{Pic} A_{K} \text {. }
$$

By Lemma 2.1, $r_{N(X), A}(\operatorname{Pic} X)$ is of finite index in Pic $A_{K}$. Thus the image $\operatorname{Im} r_{A}$ is a subgroup of finite index, of Pic $A_{K}$. Therefore, by Lemma 2.2, $\operatorname{Pic} A_{K}$ is also a finitely generated abelian group of rank $\rho(X)-\rho(Y)$. Now subgroups of Pic $A_{K}$ are all finitely generated abelian groups. So, we may speak of their rank. We denote the $\mathbb{Q}$-linear extension of $r_{A}$ by

$$
\left(r_{A}\right)_{\mathbb{Q}}:(\operatorname{Pic} X / \operatorname{Pic} Y)_{\mathbb{Q}} \longrightarrow\left(\operatorname{Pic} A_{K}\right)_{\mathbb{Q}} \text {. }
$$

$\left(r_{A}\right)_{\mathbb{Q}}$ is a surjective linear map.

Lemma 2.3. $\mathrm{MW}(\varphi)$ is a finitely generated abelian group and satisfies

$$
\operatorname{rank} \operatorname{MW}(\varphi)=\operatorname{rank} \operatorname{Pic} A_{K}-\operatorname{rank} \operatorname{NS}\left(A_{K}\right) .
$$

Proof. Since $c$ is surjective and its kernel is $\mathrm{Pic}^{0} A_{K}$, we have

$$
\operatorname{rank} \mathrm{Pic}^{0} A_{K}=\operatorname{rank} \mathrm{Pic} A_{K}-\operatorname{rank} \mathrm{NS}\left(A_{K}\right) \text {. }
$$

As well-known, $\hat{A}_{K}$ and $A_{K}$ are mutually isogenous over $K$. Thus, $\mathrm{MW}(\varphi)=$ $A(K)$ is also a finitely generated abelian group of the same rank as $\mathrm{Pic}^{0} A_{K}=$ $\hat{A}_{K}(K)$. Combining this with the formula above, we obtain the desired equality.

Lemma 2.4. (1) $\sum_{i=1}^{k}\left(m_{i}-1\right)$ divisor classes

$$
D_{i j}\left(1 \leq i \leq k, 1 \leq j \leq m_{i}-1\right)
$$

are $\mathbb{Q}$-linearly independent in $\left(\mathrm{Pic} X / \varphi^{*} \mathrm{PicY}\right)_{\mathbb{Q}}$.

(2) $\operatorname{dim} \operatorname{Ker}\left(\left(r_{A}\right)_{\mathbb{Q}}\right)=\sum_{i=1}^{k}\left(m_{i}-1\right)$.

Proof. Let us show (1). There is a large sufficiently divisible number $M$ such that $M D_{i j}$ are all Cartier. We suffice to show that there is no non-trivial relation among $1+\sum_{i=1}^{k}\left(m_{i}-1\right)$ Cartier divisor classes

$$
\varphi^{*} L(L \in \operatorname{Pic} Y), M D_{i j}\left(1 \leq i \leq k, 1 \leq j \leq m_{i}-1\right) .
$$

Since $Y$ is projective and $\mathbb{Q}$-factorial, we may assume that $L$ is the class of $H_{1}-H_{2}$, where $H_{i}$ are effective Cartier divisors. Consider the equation in Pic $X$ with integral coefficients:

$$
c \varphi^{*} H_{1}+\sum_{i=1}^{k} \sum_{j=1}^{m_{i}-1} c_{i j} D_{i j}=c \varphi^{*} H_{2} .
$$

Let $C \subset Y$ be a smooth curve which is a complete intersection of $\operatorname{dim} Y-1$ sufficiently general very ample divisors. Pulling back the above equation to the induced fibration $X_{C}=X \times_{C} Y \longrightarrow C$, one obtains the same equation as above, but with $\operatorname{dim} C=1$. Let $S \subset X_{C}$ be a normal projective surface which is a complete intersection of $\operatorname{dim} X_{C}-2$ sufficiently general very ample divisors. 
By pulling back the above equation further to the induced fibration $S \longrightarrow C$, one obtains the same equation as above, but now $\operatorname{dim} S=2$ and $\operatorname{dim} C=1$. Hence $c_{i j}=0$ by the Zariski lemma (see eg. [1, Chap. III, Lemma 8.3]). This implies $c L=0$ in $\operatorname{Pic}(Y)$ as well.

Let us show (2). It is clear that the $\mathbb{Q}$-divisor classes generated by the divisors in (1) are in the kernel. Let us show the other inclusion. The argument below is quite close to the original argument in Shioda-Tate formula (see [20, $22])$.

Let $D$ be an integral Cartier divisor on $X$ such that $\operatorname{cl}(D \mid A)=0$. It suffices to show that the linear equivalence class of $D$ is proportional to the sum of divisors in (1) modulo $\varphi^{*} \operatorname{Pic} Y$. (Indeed, $X$ is $\mathbb{Q}$-factorial.)

By $\operatorname{cl}(D \mid A)=0$, there is an element $f \in K(A) \backslash\{0\}$ such that $D \mid A=$ $\operatorname{div} f$ as divisors on $A$. Since $K(A)=\mathbb{C}(X)$ and since $K$ is the function field of $Y$, regarding $f$ as an element of $\mathbb{C}(X)$, one can also write this equality as $(D-\operatorname{div} f) \mid A=0$. This is again an equality of Weil divisors, but here we regard $\operatorname{div} f$ as a divisor on $X$. Then the support of $D-\operatorname{div} f \operatorname{does}$ not dominate $Y$. So, by replacing $D$ by a suitable linearly equivalent divisor, we may (and will) assume that $\operatorname{Supp} D$ does not dominate $Y$. By $\operatorname{Supp} D=\cup D_{l}$, we denote the irreducible decomposition of $\operatorname{Supp} D$. Since $\varphi$ is equi-dimensional in codimension 1 and $\varphi\left(D_{l}\right) \neq Y$, it follows that $\varphi\left(D_{l}\right)$ are Weil divisors on $Y$. Again, since both $X$ and $Y$ are $\mathbb{Q}$-factorial and since $\varphi$ is equi-dimensional in codimension 1 , there are positive integers $n_{l}$ and $n_{i j}^{\prime}$ such that each $n_{l} D_{l}$ is either an element of $\varphi^{*} \operatorname{Pic} Y$, or one of $n_{i j}^{\prime} D_{i j}\left(1 \leq i \leq k, 0 \leq j \leq m_{i}-1\right)$. Since $\sum_{j=0}^{m_{i}-1} c_{i j} D_{i j} \in \varphi^{*} \operatorname{Pic} Y$ for suitably chosen $c_{i j}>0$, the result follows.

By Lemma 2.4, we have

$$
\operatorname{rank} \operatorname{Pic} A_{K}=\rho(X)-\rho(Y)-\sum_{i=1}^{k}\left(m_{i}-1\right) .
$$

Substituting this into the equality in Lemma 2.3, we obtain the desired formula in Theorem 1.1.

\section{Applications for an abelian fibered hyperkähler manifold}

In this section, we shall work in the category of complex analytic spaces. Especially, unless stated otherwise, the term generic point means a closed point being in the complement of the union of countably many proper closed analytic subvarieties.

By a hyperkähler manifold we mean a compact simply-connected complex Kähler manifold $M$ having everywhere non-degenerate holomorphic 2-form $\sigma_{M}$ such that $H^{0}\left(M, \Omega_{M}^{2}\right)=\mathbb{C} \sigma_{M}$. We note that $\operatorname{dim}_{\mathbb{C}} M$ is even. 
For example, a K3 surface $S$ is a 2-dimensional hyperkähler manifold and its Hilbert scheme of 0-dimensional closed subscheme of length $d$

$$
S^{[d]}:=\operatorname{Hilb}^{d} S
$$

is a hyperkähler manifold of dimension $2 d([2])$.

By a result of Matsushita [13, 14], any abelian fibered hyperkähler manifold satisfies the conditions (1)-(4) required in Theorem 1.1 except perhaps the projectivity. See also Proposition 3.2 about the projectivity of a fibered hyperkähler manifold.

We refer to the readers an excellent account [7, Part III] for basic properties about hyperkähler manifolds.

In this section, we shall show the following:

Theorem 3.1. (1) For each positive integer $d \geq 2$ and each integer $\rho$ with $2 \leq \rho \leq 20$, there is an abelian fibered hyperkähler manifold $f_{d, \rho}: M_{d, \rho} \longrightarrow \mathbb{P}^{d}$ of dimension $2 d$ such that $\rho\left(M_{d, \rho}\right)=\rho$ and $\operatorname{rank} \mathrm{MW}\left(f_{d, \rho}\right)=\rho-2$.

(2) For each positive integer $d \geq 2$, there is an abelian fibered hyperkähler manifold $f_{d}: M_{d} \longrightarrow \mathbb{P}^{d}$ of dimension $2 d$ such that the Picard number of all smooth closed fibers are greater than 1 but the Picard number of the generic fiber over $\mathbb{C}\left(\mathbb{P}^{d}\right)$ is 1 . More geometrically, all the smooth fibers are isomorphic to $E_{\zeta}^{d}$ but the generic fiber $A=M_{\eta}$ is simple over $\mathbb{C}\left(\mathbb{P}^{d}\right)$. Here $E_{\zeta}$ is the elliptic curve of period $\zeta=e^{2 \pi i / 3}$ and $E_{\zeta}^{d}$ is the $d$-th self-product of $E_{\zeta}$.

In the rest, we shall prove Theorem 3.1.

Let us start with the following projectivity criterion due to F. Campana:

Proposition 3.2. Let $f: M \longrightarrow B$ be a surjective morphism from a hyperkähler manifold to a normal projective variety $B$ with connected fibers. Assume that $0<\operatorname{dim} B<\operatorname{dim} M=2 d$ and $M$ has a subvariety $S$ such that $f(S)=B$ and $\operatorname{dim} S=\operatorname{dim} B$. Then $M$ is projective and the generic fiber $M_{t}$ is an abelian variety.

Proof. By definition, $M$ is Kähler. By Matsushita [13], any generic fiber $M_{t}=$ $f^{-1}(t)$ is a smooth Lagrangian submanifold, i.e., $\sigma_{M} \mid M_{t}=0$ and $\operatorname{dim} M_{t}=$ $\operatorname{dim} M / 2=d$. (Note that in [13], $M$ is also assumed to be projective. However, the argument there works for non-projective $M$ if one uses a Kähler class of $M$ instead of an ample class of $M$ used there.) This implies that

$$
\Omega_{M_{t}}^{1} \simeq N_{M_{t} / M} \simeq \mathcal{O}_{M_{t}}^{\oplus d}
$$

and therefore, that $M_{t}$ is a $d$-dimensional complex torus. Moreover, by Voisin's lemma [5], $M_{t}$ is also projective. Thus $M_{t}$ is an abelian variety. Since $B$ is projective and $f(S)=B$, we have

$$
d=\operatorname{dim} B=a(B) \leq a(S) \leq \operatorname{dim} S=d .
$$

Here $a(S)$ (resp. $a(B)$ ) is the algebraic dimension of $S$ (resp. $B$ ). Thus, $a(S)=$ $\operatorname{dim} S=d$. Hence $S$ is bimeromorphic to a projective variety. In particular, 
$S$ is covered by complete algebraic curves. Thus, any two generic points in $M$ can be connected by a chain of complete algebraic curves. Therefore, $M$ is Moishezon, i.e., $\operatorname{dim} M=a(M)$, by a result of Campana [3] (see also [4] for a different approach). Since $M$ is Kähler, $M$ is then projective by Moishezon's criterion [16].

Proposition 3.3. Assume that there is an abelian fibered (projective) hyperkähler manifold $f: M \longrightarrow \mathbb{P}^{d}$ having holomorphic zero section $O$ and $r$ holomorphic sections, say $S_{i}(1 \leq i \leq r)$, which are linearly independent in $\mathrm{MW}(f)$. Then, there is an abelian fibered projective hyperkähler manifold $f^{\prime}: M^{\prime} \longrightarrow \mathbb{P}^{d}$ such that $\operatorname{dim} M^{\prime}=\operatorname{dim} M, \operatorname{rank} \mathrm{MW}\left(f^{\prime}\right)=r$, and $\rho\left(M^{\prime}\right)=r+2$.

In general, by a holomorphic section of a fiber space $\varphi: X \longrightarrow Y$, we mean a subvariety $S \subset X$ such that $\varphi \mid S: S \longrightarrow Y$ is an isomorphism. One can then identify $S$ with a holomorphic map $Y \longrightarrow X$ given by $t \mapsto(\varphi \mid S)^{-1}(t)$.

Proof. Let $u: \mathcal{U} \longrightarrow \mathcal{K}$ be the Kuranishi family of $M$, in which we assume that $0 \in \mathcal{K}, M=\mathcal{U}_{0}:=u^{-1}(0)$. Each fiber $\mathcal{U}_{t}:=u^{-1}(t)(t \in \mathcal{K})$ is a hyperkähler manifold. Let $\sigma_{t}$ be an everywhere non-degenerate holomorphic 2-form on $\mathcal{U}_{t}$. Then, by choosing a marking $\iota: R^{2} u_{*} \mathbb{Z} \simeq \Lambda \times \mathcal{K}$, we can define the period map $p: \mathcal{K} \longrightarrow \mathcal{P} ; t \mapsto\left[\iota\left(\sigma_{t}\right)\right]$. Here

$$
\mathcal{P}:=\left\{[\sigma] \in \mathbb{P}\left(\Lambda_{\mathbb{C}}\right) \mid(\sigma, \sigma)=0,(\sigma, \bar{\sigma})>0\right\} \subset \mathbb{P}\left(\Lambda_{\mathbb{C}}\right) .
$$

By the local Torelli theorem, $p$ is a local isomorphism (see eg. [7, Part III]). Thus we can (and will) identify $\mathcal{K}$ with a small open neighborhood of $p(0)$ in $\mathcal{P}$, say $W$, via $p$.

In what follows, we freely shrink $0=p(0) \in W$ whenever we need to do so.

Let $L$ be the pullback of the hyperplane on $\mathbb{P}^{d}$ by $f$. Then $f=\Phi_{|D|}$. Here $\Phi_{|D|}$ is the morphism associated with the complete linear system $|L|$. Note that $O$ and $S_{i}$ are Lagrangian submanifolds of $M$, because they are isomorphic to $\mathbb{P}^{d}$ and $\mathbb{P}^{d}$ has no non-zero global holomorphic 2 -form. Note also that $H^{2}\left(\mathbb{P}^{d}, \mathbb{Z}\right) \simeq$ $\mathbb{Z}$.

Let us consider the deformation of $M$, say $W(r)(\subset W)$, which keeps the class $[L]$ being $(1,1)$-class and the Lagrangian submanifolds $O, S_{i}(1 \leq i \leq r)$ being Lagrangian. As in Sawon [18], it follows from [14] and [23] that $W(r)$ is the intersection of $W\left(\subset \mathbb{P}\left(\Lambda_{\mathbb{C}}\right)\right)$ with $2+r$ rational hyperplanes, say $H_{j}$ $(1 \leq j \leq 2+r)$, corresponding to the required conditions on $L, O$ and $S_{i}$ $(1 \leq i \leq r)$. Note that $0 \in W(r)$.

Let $w: \mathcal{M} \longrightarrow W(r)$ be the family induced from the universal family $u: \mathcal{U} \longrightarrow \mathcal{K}=W$. By construction, the line bundle $L$ and Lagrangian submanifolds $O$ and $S_{i}(1 \leq i \leq r)$ are all extended over $W(r)$, say, $\mathcal{L}, \mathcal{O}$ and $\mathcal{S}_{i}$. It is shown by [15] (see also [18]) that $h^{0}\left(\mathcal{M}_{t}, \mathcal{L}_{t}\right)=d+1$ and $h^{i}\left(\mathcal{M}_{t}, \mathcal{L}_{t}\right)=0$ $(i \geq 1)$ for all $t \in W(r)$. Thus, $w_{*} \mathcal{L}$ is a locally free sheaf and satisfies the base change property. Thus, by the freeness of $|L|$, the linear system $|\mathcal{L}|$ is $w$-free 
and gives a fibration $\tilde{f}: \mathcal{M} \longrightarrow \mathbb{P}^{d} \times W(r)$ with holomorphic sections $\mathcal{O}, \mathcal{S}_{i}$ $(1 \leq i \leq r)$, over $W(r)$. Here, we also used the fact that $\mathbb{P}^{d}$ is rigid.

Fiberwisely, $\tilde{f}$ induces an abelian fibration $\tilde{f}_{t}: \mathcal{M}_{t} \longrightarrow \mathbb{P}^{d}$ by [13] (see also Proposition 3.2) with holomorphic sections $\mathcal{O}_{t}:=\mathcal{O} \mid \mathcal{M}_{t}$ and $\mathcal{S}_{i, t}:=\mathcal{S}_{i} \mid \mathcal{M}_{t}$ $(1 \leq i \leq r)$ for each $t \in W(r)$. By Proposition 3.2, $\mathcal{M}_{t}(t \in W(r))$ is projective. We take $\mathcal{O}_{t}$ as the origin of the Mordell-Weil group $\operatorname{MW}\left(\tilde{f}_{t}\right)$. The sections $\mathcal{S}_{i, t}$ $(1 \leq i \leq r)$ are naturally regarded as elements of $\mathrm{MW}\left(\tilde{f}_{t}\right)$.

Claim 3.4. For a generic point $t \in W(r)$, one has:

(1) $\rho\left(\mathcal{M}_{t}\right) \leq 2+r$ and

(2) $\mathcal{S}_{i, t}(1 \leq i \leq r)$ are linearly independent in the Mordell-Weil group $\operatorname{MW}\left(\tilde{f}_{t}\right)$.

Proof. By definition of generic point, we may (and will) show (1) and (2) individually.

Let us show (1). Let $l$ be the number of independent rational hyperplanes among $H_{j}(1 \leq j \leq 2+r)$. Let $V \subset W(r)$ be the set of points $t \in W(r)$ such that the number of independent element $h \in \Lambda$ satisfying that $\left(h, p\left(\sigma_{t}\right)\right)=0$ is exactly $l$.

Since each rational hyperplane in $\mathbb{P}\left(\Lambda_{\mathbb{C}}\right)$ is of the form $(h, *)=0$ for some $h \in \Lambda \backslash\{0\}$, they are countable in number. Thus $V$ is the complement of the union of countably many proper closed analytic subsets of $W(r)$.

Let $t \in V$. Then, by the Lefschetz $(1,1)$-Theorem, $\rho\left(\mathcal{M}_{t}\right)=l \leq 2+r$. This implies (1).

Let us show (2). Let $c:=\left(c_{i}\right)_{i=1}^{r} \in \mathbb{Z}^{r} \backslash\{0\}$. Consider the subset

$$
\mathcal{D}_{c}:=\left\{t \in W(r) \mid \sum_{i=1}^{r} c_{i} \mathcal{S}_{i, t}=\mathcal{O}_{t} \text { in } \operatorname{MW}\left(\tilde{f}_{t}\right)\right\},
$$

and its Zariski closure $\overline{\mathcal{D}}_{c}$ in $W(r)$. Then $\overline{\mathcal{D}}_{c}$ is a closed analytic subset of $W(r)$.

Since $\mathbb{Z}^{r} \backslash\{0\}$ is a countable set, it now suffices to show that $0 \notin \overline{\mathcal{D}}_{c}$ for each

Choose a fiber $F$ of $f=\tilde{f}_{0}: M \longrightarrow \mathbb{P}^{d}$ such that $F$ is an abelian variety, with origin $\mathcal{O} \mid F$, in which $\mathcal{S}_{i} \mid F\left(=S_{i} \mid F\right)(1 \leq i \leq r)$ are linearly independent points. By assumption, such a fiber $F$ exists. Choose then a smooth subfamily of $\tilde{f}$, say,

$$
\pi:=\tilde{f}_{\mathcal{F}}: \mathcal{F} \longrightarrow W(r)
$$

such that $F=\mathcal{F}_{0}=\pi^{-1}(0)$ and $\mathcal{F}_{t}=\pi^{-1}(t)$ is a fiber of $\tilde{f}_{t}$, which is an abelian variety with origin $\mathcal{O} \mid \mathcal{F}_{t}$. Again such a family exists. Note also that $\mathcal{O} \mid \mathcal{F}$ and $\mathcal{S}_{i} \mid \mathcal{F}$ are holomorphic sections of $\pi$. Since an abelian variety contains no rational curve, the sum $\sum_{i=1}^{r} c_{i} \mathcal{S}_{i} \mid \mathcal{F}$, as well as $\mathcal{O} \mid \mathcal{F}$ and $\mathcal{S}_{i} \mid \mathcal{F}$, defines (not only a rational section but also) a holomorphic section of $\pi$. 
Regard $\mathcal{O} \mid \mathcal{F}$ and $\sum_{i=1}^{r} c_{i} \mathcal{S}_{i} \mid \mathcal{F}$ as holomorphic maps from $W(r)$ to $\mathcal{F}$, rather than subvarieties of $\mathcal{F}$. Then, by definition of $\mathcal{D}_{c}$, one has

$$
\left(\sum_{i=1}^{r} c_{i} \mathcal{S}_{i} \mid \mathcal{F}\right)(t)=(\mathcal{O} \mid \mathcal{F})(t)
$$

for all $t \in \mathcal{D}_{c}$. Since the sections $\sum_{i=1}^{r} c_{i} \mathcal{S}_{i} \mid \mathcal{F}$ and $\mathcal{O} \mid \mathcal{F}$ are both holomorphic, we obtain

$$
\left(\sum_{i=1}^{r} c_{i} \mathcal{S}_{i} \mid \mathcal{F}\right)\left(t^{\prime}\right)=(\mathcal{O} \mid \mathcal{F})\left(t^{\prime}\right)
$$

for all $t^{\prime} \in \overline{\mathcal{D}}_{c}$. Thus $0 \notin \overline{\mathcal{D}}_{c}$. Indeed, otherwise, we would have

$$
\left(\sum_{i=1}^{r} c_{i} \mathcal{S}_{i} \mid \mathcal{F}\right)(0)=(\mathcal{O} \mid \mathcal{F})(0) \text {, i.e., } \sum_{i=1}^{r} c_{i} S_{i}|F=O| F,
$$

a contradiction to the choice of $F$.

Let us return back to the proof of Proposition 3.3. Let $t \in W(r)$ be a generic point in Claim 3.4. Then $\operatorname{rank} \operatorname{MW}\left(f_{t}\right) \geq r$. Hence $\rho\left(\mathcal{M}_{t}\right) \geq 2+r$ by Theorem 1.1. Combining this with Claim 3.4(1), we obtain $\rho\left(\mathcal{M}_{t}\right)=2+r$, and therefore, $\operatorname{MW}\left(f_{t}\right)=r$. We may now take this $\tilde{f}_{t}: \mathcal{M}_{t} \longrightarrow \mathbb{P}^{d}$ as $f^{\prime}: M \longrightarrow$ $\mathbb{P}^{d}$.

Now we are ready to prove Theorem 3.1.

Proof of Theorem 3.1. (1) As well-known (see eg. [17]), there is an elliptic K3 surface $\varphi: S \longrightarrow \mathbb{P}^{1}$ with section $O$ such that $\rho(S)=20$ and $\operatorname{rank} \operatorname{MW}(\varphi)=18$.

We have an abelian fibration

$$
\varphi_{d}: S^{[d]} \longrightarrow \mathbb{P}^{d}=\operatorname{Sym}^{d} \mathbb{P}^{1},
$$

which is the composition of the Hilbert-Chow morphism $S^{[d]} \longrightarrow \operatorname{Sym}^{d} S$ and the natural map $\operatorname{Sym}^{d} S \longrightarrow \operatorname{Sym}^{d} \mathbb{P}^{1}$ induced by $\varphi$. Moreover, the 0 -section and 18 independent sections of $\varphi$ give rise to the holomorphic 0 -section and 18 independent holomorphic sections of $\varphi_{d}$. Here we also used the fact that Hilb $^{d} \mathbb{P}^{1} \simeq \mathbb{P}^{d}$ and there is no non-trivial birational morphism from $\mathbb{P}^{d}$ to $\mathbb{P}^{d}$.

Applying Proposition 3.3 to this $\varphi_{d}$, we obtain Theorem 3.1(1).

(2) Let $f: S \longrightarrow \mathbb{P}^{1}$ be an elliptic K3 surface defined by the Weierstrass equation

$$
y^{2}=x^{3}-\left(t^{11}-1\right) .
$$

It is well-known that $\rho(S)=2$ (see eg. [12]). This follows from the fact that $S$ admits a non-symplectic automorphism $g$ of maximum order 66 :

$$
g^{*}(x, y, t)=\left(\zeta_{3} x,-y, \zeta_{11} t\right)
$$

Here $\zeta_{n}=e^{2 \pi i / n}$. Indeed, $g$ then acts on the space of global holomorphic 2 -forms as

$$
g^{*} \frac{d x \wedge d t}{y}=\zeta_{66}^{-5} \frac{d x \wedge d t}{y}
$$


This implies that

$$
20=\varphi(66) \mid \operatorname{rank} T(S)=22-\rho(S) \leq 21 .
$$

Here $\varphi$ is the Euler function and $T(S)$ is the transcendental lattice of $S$ (cf. [1]). Thus, $\rho(S)=2$. From the Weierstrass equation, we also see that each smooth fiber of $f$ is isomorphic to $E_{\zeta}$ and the singular fibers are all isomorphic to the cuspidal rational curve. Let $f_{d}: S^{[d]} \longrightarrow \mathbb{P}^{d}$ be the abelian fibration induced by $f$. Each smooth fiber of $f_{d}$ is then isomorphic to the product abelian variety $E_{\zeta_{3}}^{d}$.

Let us consider the generic fiber $A$ of $f_{d}$ in the sense of scheme. $A$ is an abelian variety defined over $K=\mathbb{C}\left(\mathbb{P}^{d}\right)$. We shall compute the rank of $\operatorname{NS}\left(A_{K}\right)$ by using Theorem 1.1.

By $\rho(S)=2$, we have

$$
\rho\left(S^{[d]}\right)=\rho(S)+1=3 .
$$

Here " +1 " comes from the exceptional divisor $E$ of the Hilbert-Chow morphism, which is generically the blow-up of the big diagonal of the Chow variety $\operatorname{Sym}^{d} S$. Observe that the divisor $f_{d}^{-1}(\Delta)$, where $\Delta$ is the big diagonal of $\mathbb{P}^{d}=\mathrm{Sym}^{d} \mathbb{P}^{1}$, consists of two irreducible components. Indeed, one component is $E$ and the other component is the proper transform of $\bar{f}_{d}^{-1}(\Delta)$, where $\bar{f}_{d}$ is the natural morphism from $\operatorname{Sym}^{d} S$ to $\operatorname{Sym}^{d} \mathbb{P}^{1}$. Then, by Theorem 1.1,

$$
0 \leq \operatorname{rank} \mathrm{MW}\left(f_{d}\right) \leq 3-1-1-\operatorname{rank} \mathrm{NS}\left(A_{K}\right)=1-\operatorname{rank} \mathrm{NS}\left(A_{K}\right) .
$$

Since $\operatorname{rank} \mathrm{NS}\left(A_{K}\right) \geq 1$, this implies that rank NS $\left(A_{K}\right)=1$. In particular, $A$ is simple, i.e., $A$ is not isogenous to the product of lower dimensional abelian varieties, over $\mathbb{C}\left(\mathbb{P}^{d}\right)$.

This completes the proof of Theorem 3.1.

It may be interesting to compare Theorem 3.1(2) with the following probably more standard:

Example 3.5. Let $f_{i}: S_{i} \longrightarrow \mathbb{P}^{1}(i=1,2)$ be two relatively minimal rational elliptic surfaces with section, such that discriminant locus $\Delta_{i} \subset \mathbb{P}^{1}$ of $f_{i}$ are disjoint. Then, as is shown by [19], $X:=S_{1} \times_{\mathbb{P}^{1}} S_{2}$ is a smooth Calabi-Yau threefold having an abelian fibration $f: X \longrightarrow \mathbb{P}^{1}$ induced by $f_{1}$ and $f_{2}$. In this example, assume further that $f_{i}$ are generic in the sense that all singular fibers are nodal rational curves and that the generic fiber $S_{i, \eta}(i=1,2)$ are not mutually isogenous. (Most cases are such cases.) Then the Picard number of any closed generic fiber $S_{1, t} \times S_{2, t}$ and the Picard number of the generic fiber $S_{1, \eta} \times \operatorname{Spec} \mathbb{C}\left(\mathbb{P}^{1}\right) S_{2, \eta}$ in the sense of scheme are all 2 . This follows from the assumption that two direct factors are not mutually isogenous (cf. [19]). 
Under the same assumption, by using Theorem 1.1, we also obtain that $\operatorname{rank} \mathrm{MW}(f)=19-1-2=16=8+8=\operatorname{rank} \mathrm{MW}\left(f_{1}\right)+\operatorname{rank} \mathrm{MW}\left(f_{2}\right)$.

\section{References}

[1] W. Barth, K. Hulek, C. Peters, and A. Van de Ven, Compact Complex Surfaces, Second edition, Springer-Verlag, Berlin, 2004.

[2] A. Beauville, Variétés Kähleriennes dont la première classe de Chern est nulle, J. Differential Geom. 18 (1983), no. 4, 755-782.

[3] F. Campana, Coréduction algébrique d'un espace analytique faiblement kählérien compact, Invent. Math. 63 (1981), no. 2, 187-223.

[4] - Reduction d'Albanese d'un morphisme propre et faiblement kahlerien. I, II, Compositio Math. 54 (1985), no. 3, 373-398; Compositio Math. 54 (1985), no. 3, 399416.

[5] _ Un critere d'isotrivialite pour les familles de varietes hyperkäleriennes sans facteur algebrique, math.AG/0408148.

[6] G. Cornell and J. H. Silverman, Arithmetic Geometry, Springer-Verlag, New York, 1986.

[7] M. Gross, D. Huybrechts, and D. Joyce, Calabi-Yau Manifolds and Related Geometries, Springer-Verlag, Berlin, 2003.

[8] M. Hindry, A Pacheco, and R. Wazir, Fibrations et conjecture de Tate, J. Number Theory 112 (2005), no. 2, 345-358.

[9] B. Kahn, Démonstration géométrique du théorème de Lang-Néron, math.AG/0703063.

[10] Y. Kawamata, Crepant blowing-up of 3-dimensional canonical singularities and its application to degenerations of surfaces, Ann. of Math. (2) 127 (1988), no. 1, 93-163.

[11] On the cone of divisors of Calabi-Yau fiber spaces, Internat. J. Math. 8 (1997), no. 5, 665-687.

[12] S. Kondō, Automorphisms of algebraic K3 surfaces which act trivially on Picard groups, J. Math. Soc. Japan 44 (1992), no. 1, 75-98.

[13] D. Matsushita, On fibre space structures of a projective irreducible symplectic manifold, Topology 38 (1999), no. 1, 79-83; Addendum: "On fibre space structures of a projective irreducible symplectic manifold", Topology 40 (2001), no. 2, 431-432.

[14] Equidimensionality of Lagrangian fibrations on holomorphic symplectic manifolds, Math. Res. Lett. 7 (2000), no. 4, 389-391.

[15] Higher direct images of dualizing sheaves of Lagrangian fibrations, Amer. J. Math. 127 (2005), no. 2, 243-259.

[16] B. Moishezon, On $n$-dimensional compact complex manifolds having $n$ algebraically independent meromorphic functions. I, Izv. Akad. Nauk SSSR Ser. Mat. 30 (1966), $133-174$.

[17] K. Oguiso, Local families of K3 surfaces and applications, J. Algebraic Geom. 12 (2003), no. 3, 405-433.

[18] J. Sawon, Deformations of holomorphic Lagrangian fibrations, math.AG/0509223.

[19] C. Schoen, On fiber products of rational elliptic surfaces with section, Math. Z. 197 (1988), no. 2, 177-199.

[20] T. Shioda, On elliptic modular surfaces, J. Math. Soc. Japan 24 (1972), 20-59.

[21] _ On the Mordell-Weil lattices, Comment. Math. Univ. St. Paul. 39 (1990), no. $2,211-240$.

[22] Mordell-Weil lattices for higher genus fibration over a curve, New trends in algebraic geometry (Warwick, 1996), 359-373, London Math. Soc. Lecture Note Ser., 264, Cambridge Univ. Press, Cambridge, 1999.

[23] C. Voisin, Sur la stabilité des sous-variétés lagrangiennes des variétés symplectiques holomorphes, Complex projective geometry (Trieste, 1989/Bergen, 1989), 294-303, London Math. Soc. Lecture Note Ser., 179, Cambridge Univ. Press, Cambridge, 1992. 
DEPARTMENT OF ECONOMICS

Keio University

4-1-1 Hiyoshi KoHOKU-KU

YOKOHAMA 223-8521, JAPAN

AND

SCHOOL OF MATHEMATiCs

Korea Institute for Advanced Study

Seoul 130-722, Korea

E-mail address: oguiso@hc.cc.keio.ac.jp 